\title{
Note on Symmetric Designs and Projective Spaces*
}

\author{
WILLIAM M. KANTOR
}

In this note we shall prove the following result.

Theorem. Let $\mathscr{D}$ be a symmetric design with

$$
v=\left(q^{d+1}-1\right) /(q-1), \quad k=\left(q^{d}-1\right) /(q-1) \quad \text { and } \quad \lambda=\left(q^{d-1}-1\right) /(q-1)
$$

for some prime power $q$ and some integer $d \geqq 2$. Then $\mathscr{D}$ is isomorphic to the design of points and hyperplanes of the desarguesian projective space $P G(d, q)$ if (and only if) $\mathscr{D}$ has an automorphism group 2-transitive on its points.

This can be regarded as an axiomatization of finite projective spaces in terms of simple combinatorial and homogeneity conditions. We note that there are many examples of symmetric designs with the above $v, k, \lambda$, even when $\lambda>1[1$, pp. 113-114].

Special cases of this theorem are found in [3], $\S \S 9-10$. The present proof is quite different from and much simpler than those of [3]. We remark that the following argument can be refined to give some information concerning 2 -transitive symmetric designs in which $k-\lambda$ is a power of a prime not dividing $v$.

Let $\mathscr{D}$ be as in the theorem and set $\Gamma=$ Aut $\mathscr{D}$. The line $x y$ determined by a pair of distinct points $x, y$ is the intersection of all the blocks containing $x$ and $y$. All lines have the same number $m+1$ of points. There is a unique line containing two given distinct points $[1, \mathrm{p} .65]$.

By the Ostrom-Wagner theorem [4] we may assume that $\lambda>1$. Let $q=p^{e}$ with $p$ a prime. If $B$ and $C$ are distinct blocks and $x \in B \cap C$ there are $(k-1) / m$ lines in $B$ through $x$ and $(\lambda-1) / m$ lines in $B \cap C$ through $x$. Then $m \mid k-\lambda=q^{d-1}$. Also, $m+1 \leqq(v-\lambda) /(k-\lambda)=q+1 \quad[1$, p.65]. By the Dembowski-Wagner theorem [2] our result will hold if $m=q$. We have $m=p^{f}$ with $f \leqq e$.

Since $(v, k)=1$, by $\left[1\right.$, p. 79] if $x \neq y$ then $\Gamma_{x y}$ is transitive on the blocks on $x$ not on $y$. There are $k-\lambda=q^{d-1}$ such blocks. It follows that a Sylow $p$-subgroup $\Sigma$ of $\Gamma_{x y}$ is also transitive on the blocks on $x$ not on $y$.

$\Sigma$ acts on the $v-(m+1)$ points not in $x y$. Here

$$
v-(m+1)=q\left(q^{d}-1\right) /(q-1)-m \text {. }
$$

Assume that $m<q$. Then $p m \nmid v-(m+1)$. There is thus a point $z \notin x y$ such that $\left|z^{\Sigma}\right|=p^{i} \leqq m$. Then

$$
\left|B^{\Sigma_{z}}\right|=\left|\Sigma_{z}: \Sigma_{z B}\right|=\left|\Sigma: \Sigma_{B}\right|\left|\Sigma_{B}: \Sigma_{z B}\right| /\left|\sum: \Sigma_{z}\right| \geqq q^{d-1} / p^{i} \geqq q^{d-1} / m
$$

\footnotetext{
* Research supported in part by NSF Grant GP 9584
} 
for each block $B$ on $x$ not on $y$. Since $y \notin x z$ we can choose $B$ so that $x, z \in B$ and $y \notin B$. Then each block in $B^{\Sigma_{z}}$ is on $x$ and $z$. Thus,

$$
\left(q^{d-1}-1\right) /(q-1)=\lambda \geqq\left|B^{\Sigma_{z}}\right| \geqq q^{d-1} / m=p^{e-\delta} q^{d-2} \geqq p q^{d-2},
$$

which is impossible.

Consequently, $m=q$, which proves the theorem.

\section{References}

1. Dembowski, P.: Finite geometries. Berlin-Heidelberg-New York: Springer 1968.

2. - Wagner, A.: Some characterizations of finite projective spaces. Arch. der Math. 11, 465-469 (1960).

3. Kantor, W. M.: 2-Transitive symmetric designs. Trans. Amer. Math. Soc. 146, 1-28 (1969).

4. Ostrom, T. G., Wagner, A.: On projective and affine planes with transitive collineation groups. Math. Z. 71, 186-199 (1959).

\author{
Dr. William M. Kantor \\ Department of Mathematics \\ University of Illinois \\ Chicago, Illinois 60680 \\ USA
}

